\title{
AN IDENTITY FOR CERTAIN DIRICHLET SERIES $\dagger$
}

\author{
by B. C. BERNDT
}

(Received 16 March, 1967)

1. Introduction. In deriving the approximate functional equation for certain Dirichlet series, one first establishes an identity for the function in terms of a partial sum of the series (e.g. see [1] and [2]). It is the purpose of this note to give a short proof of this identity for Hecke's Dirichlet series [1]. The proof is valid with only a few minor changes for the identity given by Chandrasekharan and Narasimhan [2, Lemma 2] for a much larger class of Dirichlet series. However, the brevity of the paper would be lost if we introduced the necessary terminology and notation.

2. Notation and preliminary results. In the sequel, $s=\sigma+i$ with $\sigma$ and $t$ both real. The summation sign $\sum$ appearing with no indices will always mean $\sum_{n=1}^{\infty}$. If $c$ is real, we denote the integral

$$
\int_{c-i \infty}^{c+i \infty} \text { by } \int_{(c)} \text {. }
$$

We shall need the following well known estimates:

$$
\Gamma(s) \sim(2 \pi)^{\frac{1}{2}}|t|^{\sigma-\frac{1}{2}} e^{-\frac{1}{2} \pi|t|},
$$

as $|t| \rightarrow \infty$; if $J_{v}(x)$ denotes the usual Bessel function of order $v$,

$$
J_{v}(x) \sim\left(\frac{2}{\pi x}\right)^{\frac{1}{2}} \sum_{n=0}^{\infty} \frac{c_{n} e^{i x}+c_{n}^{\prime} e^{-i x}}{x^{n}},
$$

as $x \rightarrow \infty$, where $c_{n}$ and $c_{n}^{\prime}(0 \leqq n<\infty)$ are constants.

Also [4, p. 196], for $x>0$,

$$
J_{v}(x)=\frac{1}{2 \pi i} \int_{(\mathrm{c})}\left(\frac{x}{2}\right)^{v-2 s} \frac{\Gamma(s)}{\Gamma(v+1-s)} d s \quad\left(0<c<\frac{1}{2} \operatorname{Re} v+\frac{3}{4}\right) .
$$

Lemma 1. Let $\sigma<\frac{1}{2}$ and $t$ be fixed. Then

as $\xi \rightarrow \infty$.

$$
\int_{\xi}^{\infty} u^{\sigma+i t} J_{v}(u) d u=O\left(\xi^{\sigma-\frac{1}{2}}\right)
$$

Proof. By (2), it is sufficient to examine

$$
\int_{\xi}^{\infty} u^{\sigma-\frac{1}{2}+i t} e^{i u} d u
$$

An integration by parts easily yields the result.

$\dagger$ The main result of this paper appeared in the author's Ph.D. thesis written at the University of Wisconsin in 1966 under the direction of Professor J. R. Smart. 
LemMa 2. Let $q$ be a positive integer and suppose that $0<c<\sigma$. Then

$$
\frac{1}{2 \pi i} \int_{(c)} \frac{\Gamma(s+q) \Gamma(w) x^{w-s}}{\Gamma(s) \Gamma(w+q)(s-w)} d w=\left\{\begin{array}{cc}
1 & \text { if } 0<x<1, \\
\frac{\Gamma(s+q)^{q-1}}{\Gamma(s)} \sum_{j=0} \frac{(-1)^{j} x^{-j-s}}{j ! \Gamma(q-j)(s+j)} & \text { if } x \geqq 1 .
\end{array}\right.
$$

Proof. Choose $T$ so that $|s|<R, q<R$, where $c^{2}+T^{2}=R^{2}$. Let $L$ denote $(c-i T$, $c+i T), C_{1}$ that part of $|w|=R$ to the left of $L$, and $C_{2}$ that part of $|w|=R$ to the right of $L$.

For $x<1$ we have

$$
\frac{1}{2 \pi i} \int_{c_{2}-L} \frac{\Gamma(s+q) \Gamma(w) x^{w-s}}{\Gamma(s) \Gamma(w+q)(s-w)} d w=-1
$$

The integral over $C_{2}$ clearly tends to 0 as $R \rightarrow \infty$, since $q \geqq 1$. The result for $x<1$ follows.

For $x \geqq 1$ consider

$$
\frac{1}{2 \pi i} \int_{c_{2}+L} \frac{\Gamma(s+q) \Gamma(w) x^{w-s}}{\Gamma(s) \Gamma(w+q)(s-w)} d w
$$

$\Gamma(w) / \Gamma(w+q)$ has a simple pole at $w=-j$ with residue $(-1)^{j} /\{j ! \Gamma(q-j)\}$ for $0 \leqq j \leqq q-1$. The integral over $C_{1}$ tends to 0 as $R \rightarrow \infty$, and the result follows.

3. THEOREM. Let $\phi$ be a Dirichlet series of signature $(\lambda, r, \gamma)$ with abscissa of absolute convergence $\sigma_{a}$ and residue $\rho$ at $s=r$. Let $q$ be a positive integer,

$$
R_{0}=\frac{\Gamma(s+q) \phi(0) x^{-s}}{\Gamma(s) \Gamma(q) s}, \quad R_{r}=\frac{\Gamma(s+q) \Gamma(r) \rho x^{r-s}}{\Gamma(s) \Gamma(r+q)(s-r)},
$$

and $\xi=4 \pi(n x)^{\frac{1}{2}} / \lambda$. Then, for $x>0, \sigma>\frac{1}{2}\left(r-q-\frac{1}{2}\right)$, and $q>2 \sigma_{a}-r-\frac{1}{2}$,

$$
\begin{aligned}
\phi(s)=\sum_{n \leqq x} a(n) n^{-s}-\frac{\Gamma(s+q) x^{-s}}{\Gamma(s)} & \sum_{n \leqq x} a(n) \sum_{j=0}^{q-1} \frac{(-n / x)^{j}}{j ! \Gamma(q-j)(s+j)}+R_{0}+R_{r} \\
& +\gamma \frac{\Gamma(s+q)}{\Gamma(s)}\left(\frac{2 \pi}{\lambda}\right)^{2 s-r} \sum \frac{a(n)}{n^{r-s}} \int_{\xi}^{\infty}\left(\frac{u}{2}\right)^{r-q-2 s} J_{r+q-1}(u) d u .
\end{aligned}
$$

Proof. For $\sigma_{a}<c<\sigma$,

$$
\frac{1}{2 \pi i} \int_{(c)} \frac{\Gamma(s+q) \Gamma(w) \phi(w) x^{w-s}}{\Gamma(s) \Gamma(w+q)(s-w)} d w=\sum_{n>x} a(n) n^{-s}+\frac{\Gamma(s+q) x^{-s}}{\Gamma(s)} \sum_{n \leqq x} a(n) \sum_{j=0}^{q-1} \frac{(-n / x)^{j}}{j ! \Gamma(q-j)(s+j)}
$$

upon an application of Lemma 2 . Since $q \geqq 1$, the change in order of summation and integration is justified by absolute convergence.

We now move the line of integration to $r-\sigma_{a}-\varepsilon+i t(\varepsilon>0,-\infty<t<\infty)$, by integrating around a rectangle with vertices $c \pm i T$ and $r-\sigma_{a}-\varepsilon \pm i T$ and then letting $T \rightarrow \infty$. By a Phragmén-Lindelöf theorem, the integrals along the horizontal edges tend to 0 as $T \rightarrow \infty$ if $q>2 \sigma_{a}-r-1$. Thus, since $\phi(-k)=0(k=0,1,2, \ldots)$,

$$
\frac{1}{2 \pi i} \int_{(c)} \frac{\Gamma(s+q) \Gamma(w) \phi(w) x^{w-s}}{\Gamma(s) \Gamma(w+q)(s-w)} d w=\frac{1}{2 \pi i} \int_{\left(r-\sigma_{a}-\varepsilon\right)} \frac{\Gamma(s+q) \Gamma(w) \phi(w) x^{w-s}}{\Gamma(s) \Gamma(w+q)(s-w)} d w+R_{0}+R_{r} .
$$


Denote the first term on the right-hand side of $(6)$ by $I(s, x, q)$. In examining $I(s, x, q)$ we shall regard $q$ as a complex variable. Replacing $w$ by $r-w$, using the functional equation for $\phi$, and inverting the order of summation and integration by absolute convergence if $\operatorname{Re} q>2 \sigma_{a}-r$, we find that

$$
I(s, x, q)=\gamma \frac{\Gamma(s+q)}{\Gamma(s)}\left(\frac{2 \pi}{\lambda}\right)^{r} x^{r-s} \sum a(n) \frac{1}{2 \pi i} \int_{\left(\sigma_{a}+\varepsilon\right)}\left(\frac{\xi}{2}\right)^{-2 w} \frac{\Gamma(w)}{\Gamma(r+q-w)(s-r+w)} d w .
$$

Now, from (3),

$$
u^{-r-q+1} J_{r+q-1}(u)=\frac{1}{2 \pi i} \int_{(c)} \frac{2^{2 w-r-q+1} \Gamma(w) u^{-2 w}}{\Gamma(r+q-w)} d w \quad\left(0<c<\frac{1}{2}(r+\operatorname{Re} q-1)+\frac{3}{4}\right) .
$$

Multiply both sides by $u^{2 r-1-2 s}$ and integrate over $(\xi, \infty)$. Using a standard theorem [3, p. 349] to invert the order of integration we find that

$$
\int_{\xi}^{\infty} u^{r-q-2 s} J_{r+q-1}(u) d u=\frac{1}{2 \pi i} \int_{(c)} \frac{2^{2 w-r-q} \Gamma(w) \xi^{2(r-s-w)}}{\Gamma(r+q-w)(s-r+w)} d w
$$

provided that $\sigma>r-c, \operatorname{Re} q>2 c-r$, and $\sigma>\frac{1}{2}\left(r-\operatorname{Re} q-\frac{1}{2}\right)$. Substituting (8) into (7), we obtain

$$
I(s, x, q)=\gamma \frac{\Gamma(s+q)}{\Gamma(s)}\left(\frac{2 \pi}{\lambda}\right)^{2 s-r} \sum \frac{a(n)}{n^{r-s}} \int_{\xi}^{\infty}\left(\frac{u}{2}\right)^{r-q-2 s} J_{r+q-1}(u) d u
$$

provided that $\sigma>r-\sigma_{a}, \operatorname{Re} q>2 \sigma_{a}-r$, and $\sigma>\frac{1}{2}\left(r-\operatorname{Re} q-\frac{1}{2}\right)$. However, by Lemma 1,

$$
I(s, x, q)=O\left(x^{\frac{\hbar}{2(r-R e q-t)}}\right),
$$

if $\operatorname{Re} q>2 \sigma_{a}-r-\frac{1}{2}$. Therefore, $I(s, x, q)$ converges absolutely if $\operatorname{Re} q>2 \sigma_{a}-r-\frac{1}{2}$, and by analytic continuation (9) is valid for $\operatorname{Re} q>2 \sigma_{a}-r-\frac{1}{2}$. Once again restricting ourselves to positive integral values for $q$ and combining (5), (6) and (9), we have established (4) for $q>2 \sigma_{a}-r-\frac{1}{2}$. By analytic continuation (4) is valid for $\sigma>\frac{1}{2}\left(r-q-\frac{1}{2}\right)$, and the proof is complete.

Suppose that $\phi$ has signature $(1, r, \gamma)$ and is entire. Then it is well known that $\sigma_{a} \leqq \frac{1}{2}(r+1)$, and so we may take $q=1$ in (4). Hence, by (10), for $\sigma>\frac{1}{2} r-\frac{3}{4}$,

$$
\phi(s)=\lim _{x \rightarrow \infty}\left(\sum_{n \leqq x} a(n) n^{-s}-x^{-s} \sum_{n \leqq x} a(n)\right)
$$




\section{REFERENCES}

1. T. M. Apostol and Abe Sklar, The approximate functional equation of Hecke's Dirichlet series, Trans. Amer. Math. Soc. 86 (1957), 446-462.

2. K. Chandrasekharan and Raghavan Narasimhan, The approximate functional equation for a class of zeta-functions, Math. Ann. 152 (1963), 30-64.

3. E. W. Hobson, The theory of functions of a real variable, vol. II, 2nd. ed., Cambridge University Press (Cambridge, 1926).

4. E. C. Titchmarsh, Theory of Fourier integrals, 2nd. ed., Clarendon Press (Oxford, 1948).

The University of Glasgow

and

THE UNIVERSITY OF IllinoIS 